\title{
Differences between ethnic and non ethnic-specific clinics for Portuguese-speaking mental health patients explained by providers
}

\author{
Marta Gonçalves ${ }^{1,2}$, Diana Farcas ${ }^{1}$ and Benjamin Cook ${ }^{2}$
}

\begin{abstract}
Background: A previous quantitative study conducted in a health care system in a Northeastern U.S. metropolitan area identified greater adequacy of mental health care for Portuguese-speaking patients at a ethnic-specific Portuguese Mental Health Program (PMHP) compared to non ethnic-specific clinics. The objective of the present study was to understand, from a provider perspective, the disadvantages and difficulties of treating Portuguese-speaking immigrants with mental illness, and to elicit recommendations for improving care for this population.
\end{abstract}

Methods: We conducted three interviews with providers using a structured interview guide that elicited questions related to the clinic, its patients, provider's work, and ways in which the providers tailored mental health services to the Portuguese-speaking population. Responses were analyzed using content analysis, recording the frequency and saliency of particular words and phrases, and identifying keywords or repeated ideas.

Results: Providers reported that the PMHP clinic is successful because it offers a unique set of services, provides services in the Portuguese language $100 \%$ of the time, and has existed for a long time in their community. Important differences between patients from Portugal and patients from Brazil include demographic characteristics, and patients' and relatives' feelings about seeking mental health treatment.

Conclusion: This study supports policy recommendations to expand the availability of ethnic specific clinics. These clinics may play an especially important role in cost reduction and quality enhancement efforts being undertaken in urban safety net hospitals that serve a large number of individuals of racial/ethnic minority background.

Keywords: Retention, Mental health care, Ethnic specific, Qualitative, Providers perspective.

'Lisbon University Institute/ Instituto Universitário de Lisboa (ISCTE-IUL), Cis-IUL, Lisboa, Portugal

2Harvard Medical School, Cambridge Health Alliance, Cambridge, MA, USA
Citation: Gonçalves et al. Differences between ethnic and non ethnic-specific clinics for Portuguese-speaking mental health patients explained by providers. IJCNMH 2014; 1:13

Received: 18 Jul 2014; Accepted: 27 Oct 2014; Published: 05 Nov 2014

Correspondence: Marta Gonçalves

Instituto Universitário de Lisboa (ISCTE-IUL), Cis-IUL

Avenida das Forças Armadas, 1649-026 Lisboa, Portugal

Email address: marta.goncalves@iscte.pt

Open Access Publication Available at http://ijcnmh.arc-publishing.org

(C) 2014 Gonçalves et al. This is an open access article distributed under the Creative Commons Attribution License, which permits unrestricted use, distribution, and reproduction in any medium, provided the original work is properly cited. 


\section{Introduction}

Improving quality of mental health care is necessary. Prior studies have documented that racial/ethnic minorities experience greater persistence and severity of mental illness compared to whites and that there are racial/ethnic disparities in access and quality of mental health care [1-3]. Ethnic concordance between patients and clinicians contributes to quality of care by improving communication, especially during mental health intake visits [4]. In the 1990s, ethnic specific programs were found to increase return rates and length of mental health treatment [5]. In order to identify if these programs continue to improve service, a previous quantitative study compared the adequacy of care, emergency room (ER), and inpatient mental health care use of Portuguese-speaking patients receiving care at an ethnic-specific clinic, the Portuguese Mental Health Program (PMHP), with Portuguese-speaking patients receiving care in non-ethnic-specific clinics [6]. This study, conducted in a Northeastern United States urban health care system, identified improved rates of adequate care in the PMHP compared to other clinics, and concluded that policymakers should consider expanding the availability of ethnic specific clinics. In order to better understand the factors that make ethnic-specific clinics successful, and to provide concrete information for clinicians and policymakers, this paper intends to present an exploratory qualitative study based on three interviews conducted with providers at the PMHP.

\section{Objectives}

The objective of this study was to understand, from a provider perspective, the disadvantages and difficulties of treating Portuguese-speaking immigrants with mental illness, and to elicit recommendations for improving care for this population. The qualitative study complements the data obtained in the previous quantitative study. Our focus is on care that is tailored by culture and language to Portuguese speakers primarily from Brazil, Portugal, and Cape Verde.

\section{Methods}

In order to reach the proposed objectives, a qualitative study with a descriptive approach was conducted.

\section{Participants}

The participants of this study were providers currently integrated in the PMHP team. During a team meeting the original quantitative study results were presented to all providers and all were invited to participate in the follow-up qualitative study. Afterwards, each provider was contacted by email and again invited to participate in the study. Three providers participated in this study. Two participants were male and one participant was female. Participants were between the ages of 40-59 years old. The professions of the three participants were clinical social worker, social assistant/therapist, and physician. Two of these professionals worked in the PMHP, whereas the third worked in both the PMHP and in other clinics in the health care system.

\section{Instruments}

We used an interview guide and a socio-demographic questionnaire, both specifically developed for the study. The semi-structured interview guide was developed based on the literature and the objectives of the study, resulting in three groups of questions: a) eight questions related to the clinic and the patients that use mental health services; b) four questions about the provider's work at the clinic; and c) four questions related to the mental health services and recommendations for improving them.

In the first group of questions we asked:

1. What types of mental health treatment are available for the patients at this clinic?

2. How are services provided at this clinic different from services offered by other clinics?

3. a) How do patients become aware of this clinic? b) One of the results that we found in the previous study is that patients at PMHP are older than those at other clinics [in our health care system]. Why do you think this is happening?

4. What are the main reasons that encourage Portuguese speaking patients to use services at this clinic?

5. How do you consider that the patients feel about seeking mental health treatment?

6. How do you think that a patient's family/friends feel about him/her seeking mental health treatment?

7. How do you consider that patients may feel about going to a clinic known for providing mental health treatment?

8. What are the main reasons that could prevent Portuguese speaking patients from using the mental health services at this clinic?

In the second group of questions we asked:

1. How do you describe your relationship with the patients? (Can you give some positive aspects of this relationship? Can you mention something that you think it can be improved?)

2. What are the strategies used while working with the Portuguese speaking patients?

3. How would you describe your level of satisfaction with this clinic as a place to treat Portuguese speaking patients

4. If you had the opportunity to change something, what would you like to change about your own practice as a clinician working with Portuguese speaking patients?

In the third group we asked:

1. How well do you think that the PMHP is known by the Portuguese immigrant community? 
2. How well do you think that the PMHP is integrated/outreached into the Portuguese immigrant community?

3. What do you think is lacking in the mental health services system that would better serve Portuguese speaking immigrants?

4. What recommendations would you have for clinics that want to improve their treatment for Portuguese speaking patients?

Participants were also asked to report their age, clinical profession, and their affiliation with the PMHP and other clinics in the health care system.

\section{Procedure}

Participants were recruited through the PMHP clinic in collaboration with the PMHP director and other team members. Data collection was conducted in individual interviews via video-conference (Skype ${ }^{\mathrm{rm}}$, Skype Communications S.A.R.L, Luxembourg) with an average duration of 45 minutes.

We adopted a qualitative approach. Since this study intended to understand perceptions, the individual interviews aimed to explore different opinions and representations of the same subject [7]. After transcribing the interviews, they were subjected to content analysis, following Mayring's [8] reduction method. For each of the 17 questions we have reduced the answer material for each of the three participants. The system of categories was constructed retrospectively by two independent investigators [9]. The process of reducing the answer material of each of the three participants was done independently and then the two investigators met, in order to reach consensus about key organizing topics. The whole process of data collection and analysis followed the American Psychological Association (APA) ethical standards, having all participants signed informed consent forms [10].

4] the Trail Making Test [55]; the Verbal Fluency Test [55]; and the Tower Test [55].

\section{Results}

The content analysis indicated that the data could be grouped into four main themes: a range of PMHP clinic offerings to match patient preferences, enabling access to PMHP clinic, PMHP care quality, and integration of PMHP into the Portuguese speaking community.

Each one of these themes divides itself into categories, PMHP clinic offer: types of mental health treatment available, uniqueness of services provided; Acess to PMHP clinic: patient awareness of the clinic, reasons for older age of patients at PMHP, main reasons of clinic use, patients own feelings seeking mental health treatment, family/friends feelings about patient seeking mental health treatment, patient barriers to mental health services; PMHP care quality: strengths of provider-patient relationship, weaknesses of provider-patient relationship, intervention strategies, level of satisfaction, opportunities for change; PMHP among Portuguese speaking community: knowledge, integration/ outreach, mental health services system lacks for Portuguese speaking immigrants, recommendations for clinics to improve treatment for Portuguese speaking patients.

In order to obtain a clear comprehension of the analyzed data, each of them will be presented individually with its correspondent categories.

\section{A range of PMHP clinic offerings to suit the preferences of patients}

The types of mental health treatment available at PMHP for children, adolescents, adults by a small group of providers are psychopharmacological consultation, ongoing treatment, psychotherapy, specialized services (family treatment, eye movement desensitization and reprocessing (EMDR), cognitive behavioral therapy (CBT), dialectic behavior therapy (DBT), trauma services), couple and family therapy, and groups, although the last ones are, according to providers, difficult to organize because people are afraid of being in the same room with others from the community (they fear the stigma related to mental health issues). PMHP uniqueness of services provided is related to the social, cultural, and linguistic aspect (i.e. 100\% use of Portuguese language): "Well, it makes a difference to have providers speaking Portuguese or having interpreters. This makes a big difference for patients that feel much more comfortable speaking in their own language. Also, the clinic has been there for a long time and it's creating a culture, a safe environment for the members of this community. They feel more safe here. Some of them may have their primary care at a different clinic but they prefer to keep mental health care here. The primary care provider may have the service there, but they don't want to be assisted there, even with an interpreter they prefer to continue coming to us. So, it is created sort of a culture in community, in which patients feel comfortable coming to this center."

\section{Enabling Access to PMHP clinic}

Sources for patient awareness of the clinic are word of mouth, location in the community and its long history/ well known and reference by CHA. The older age of patients at $P M H P$ is, according to the providers, especially true for the Portuguese communities, not for Brazilian: first generation Portuguese immigrants arriving in the U.S. 30 years ago speak only Portuguese, whereas second generation immigrants are more likely to be seen in other non-ethnic-specific clinics. Conversely, the Brazilian community has a constant influx of immigrants every year and many Brazilians change to other towns in the metropolitan area. The average age of Brazilians is increasing to approximately 30 years old as older Brazilians enter the area. There are three main reasons of clinic use: language/culture, clinic age/ knowledge of staff (e.g. secretary)/sense of small community, and acceptance of all health insurance types. 
Concerning patients own feelings seeking mental health treatment, while the Brazilian community is much more open to seeking mental health services, in the Portuguese community there is more stigma and it is probably more difficult to engage in treatment. Portuguese males have even more difficulty in accessing services compared to female counterparts, because of stigma. Though, according to providers, tension/fear/taboo decreases when they notice that providers speak Portuguese. Parents seek treatment for their children in the same places where they seek services for themselves.

Concerning family/friends feelings about patient seeking mental health treatment, patients have stopped medications because family members tell them that they should and describe medications as "only for crazy people". According to providers there is bias in society in general against mental health treatment: although the taboo is decreasing there is still prejudice. On the other hand, there are many families that do support their patients, especially in the Brazilian population.

In respect to patients barriers/facilitators to mental health services one of the advantages is that the primary care department and mental health care services are located in the same building. This contributes to the destigmatization of seeking mental health services. As previously mentioned, group therapy sessions are difficult to set up. For some people there is still stigma, taboo, and lack of knowledge about therapy process. Another barrier is distance, "as they are immigrants they are afraid of driving without license". Another is schedule flexibility as their priority is to work "though we try to have time in the evening and in the morning". Another barrier is that "first is physical health and mental health is at the end".

\section{PMHP care quality}

Regarding strength of provider-patient relationship, providers emphasize that "parents are very happy when they meet an American who can speak Portuguese and understand them. This helps people feel comfortable and when they start talking they don't stop... And I think [people feel comfortable] also because I'm familiar with Brazilian culture; I lived there for a couple of months". One provider states doing community psychiatric requires being "really close in the way you interact with them and their families. So you feel pretty embedded into their community".

Concerning weaknesses of provider-patient relationship there is a need in the rest of the health care system for more Portuguese speaking therapists "our ratio of patients with some medication having therapy is not great". Many times the relationship gets worse because patients call to cancel the appointment and the information does not pass.

In terms of intervention strategies providers mention the adaptation of vocabulary and language to the patients' generation "so you can adapt a little bit to their knowledge about mental health, about illness, about treatment, so you have to adapt in accordance to where they came from and understand how much information they can process. So you have to speak in very simple terms or really adapt your language so they can understand it. Younger generations might have grown up being more aware of what mental illness is, but for older folks, the ones we see from the islands, this is something exoteric and magical and you really have to put it in terms where it is very specific, in a way that they can understand it." Others mention the sharing of knowledge about their culture. Others mention they could give patients literature about mental diagnosis that is translated into Portuguese. Others mention the importance of working with immigrant parents to explain strategies for better parenting.

Concerning providers' level of satisfaction, all mention an high level: "working with Portuguese immigrants... they are grateful for this. It is the sense of practicing community psychiatry."; "I identify myself a lot with the work group and patients"; "is the only clinic where I work that has a mental health team, so I feel like I get a lot of support, especially with how they try to integrate mental health services with clinic services... I feel like the entire clinic is aware of what I do, and so it just makes things easier".

In terms of opportunities for change, providers mentioned the need for having more support in the clinic (e.g. case management), more spaces for group therapy, the capacity to provide appointments every week, less paper work, the need to work there more than one day, and for continued use of long term therapy.

\section{Integrating PMHP into the Portuguese speaking community}

Concerning knowledge on PMHP, providers agree that it is very well known "mostly because of the community being very tight", "because of location", and "because there are some radios in Portuguese or Brazilian that patients comment... we could even use them to advertise our services".

Regarding integration/outreach there are flyers in the area explaining that the health care system offers services to different communities, there is a Portuguese speaker advocacy organization nearby and "we regularly refer patients to them and vice versa". One provider mentions that increasing the amount of flyers in Portuguese at the Portuguese Consulate, restaurants, and churches ("Some churches are more open to mental health") would help. Another mentions word of mouth. One provider emphasizes that they should go to the other cities where the health care system is so that people are aware and have access to PMHP.

Concerning mental health services system lacks for Portuguese speaking immigrants: the providers mentioned the need for more groups offered in Portuguese for group therapies, more case managers, specific therapies, more qualified Portuguese speaking providers, more mental health education work (e.g. how do people access this service), couple and family therapy in Portuguese in other locations, more partner work mental health provider, and more medical staff. 
Regarding recommendations for clinics to improve treatment for Portuguese speaking patient, providers mention "I think that what's offered in the clinic covers most of what's needed in the community. I mean there are other communities and other areas, where not even English speakers get access to so many services."; "we have a couple of evenings... I think we give enough flexibility to them for that" and "some services that give transportation are not for undocumented patients and others don't cover all the areas. And many of our patients are undocumented and that's a limitation"; "We have longer than average follow-up"; "we serve plenty of the population who would otherwise be uninsured." Others mentioned the importance of having providers who speak the language and understand the culture/ emigration process, and translated materials. "Honestly at this point I feel like kids who are growing up, kids who were born here into Portuguese speaking families should be encouraged to become psychologists, because the community really needs help... I remember reading about a study that said something like $50 \%$ of the visit to primary care is mental issues anyway, so..."

\section{Discussion}

The aim of this research was to understand, from a provider perspective, the disadvantages and difficulties of treating Portuguese-speaking immigrants with mental illness, and to elicit recommendations for improving care for this population. The findings complement a previous study that identified greater adequacy of care for Portuguese-speaking patients at the PMHP compared to non-ethnic-specific clinics [6]. Providers report that the PMHP clinic offers a unique set of services and that the success of the clinic services is largely due to their $100 \%$ use of Portuguese language and the clinic's longtime existence in their community. Providers reported important differences between patients from Portugal and Brazil, including Brazilian patients' younger age and the greater openness of Brazilian patients and their close relatives to seeking mental health treatment.

The results from this study suggest a number of reasons why the PMHP may improve quality of mental health care and increase retention and adequacy of treatment for Portuguese-speaking patients over and above other clinics in the same health care system. Many of the reasons cited for high quality care at the PMHP could be incorporated into clinics regardless of whether or not they have an ethnic-specific focus. Providers reported that the greater quality of care delivered at the PMHP is due largely to the strength of the provider-patient relationships. Also, they cited the diversity of treatments that they provided to the community as key to improving retention and tailoring to the preferences and needs of the patients. They recommended that other clinics improve treatment for Portuguese speaking patients by instituting more flexible hours and transportation for all independently of legal documents or insurance, and policies to improve long-term follow-up.
Other factors that contributed to quality of care were unique to the ethnic-specific clinic setting. The clinic was well known to the community and invested significant resources into hiring Portuguese-speaking clinicians and staff members, translating all written materials, and having providers with a deep understanding of the culture/ emigration process. Provider recommendations included increasing the number of Portuguese speaking therapists and improving communication across clinics and hospitals within the system.

With health care reform in the U.S. has come an emphasis on placing more and more risk on provider organizations to manage patient care, via capitated payments for patients and other incentives implemented through accountable care organizations (ACOs) and other pilot projects that move away from fee for service payment models. Ethnic-specific clinics provide a potentially important alternative for ACOs and provider organizations trying to manage the care of high-risk, racial/ethnic minority mental health patients. Demonstrated gains in adequacy of care and the greater retention and satisfaction of care for patients in these settings is likely to decrease the probability that patients will leave provider networks (potentially making ACOs responsible for higher out of network payments), and reduce long-term expenditures related to management of chronic comorbid physical illnesses and expensive hospital-based mental health care.

Previous studies have identified that ethnic-specific clinics are effective [5] and that patient-provider concordance is helpful to improving quality of care and patient satisfaction [4]. We add to this literature by presenting providers' opinions and by detailing the primary strategies of action used by providers in ethnic-specific clinics in their everyday professional life. This study has some limitations such as the small number of participants and the fact that the data were only collected in PMHP. The study, in combination with the previous quantitative study, thus adds to the preliminary evidence supporting policy recommendations to expanding the availability of ethnic specific clinics as these programs continue to improve service. The study also provides further evidence of the importance of tailoring treatment to match the language and cultural norms of racial/ethnic minority community members.

\section{Abbreviations}

ACO: Accountable care organization; APA: American Psychological Association; CBT: Cognitive behavioral therapy; DBT: Dialectic behavior therapy; EMDR: Eye movement desensitization and reprocessing; ER: Emergency room; PCP: ???; PMHP: Portuguese Mental Health Program.

\section{Competing interests}

The authors declare no conflict of interest.

\section{References}

1. Bauer, A.M., Alegria, M. Impact of patient language proficiency and interpreter service use on the quality of psychiatric care: A systematic review. Psychiatric Services 2010; 61(8):765-773 
2. Alegria, M., Sribney, W., Perez, D., Laderman, M., Keefe, K. The role of patient activation on patient-provider communication and quality of care for US and foreign born Latino patients. Journal of General Internal Medicine 2009; 24(3):534-541.

3. Pippins, JR., Alegría, M., Haas, JS. Association between language proficiency and the quality of primary care among a national sample of insured Latinos. Medical Care 2007; 45(11):1020-1025.

4. Alegría, M., Roter, DL., Valentine, A., Chen, CN., Li, X., Lin, J., Rosen, D., Lapatin, S., Normand, S., Larson, S., Shrout, P. Patientclinician ethnic concordance and communication in mental health intake visits. Patient education and counseling 2013; 93(2):188-196.

5. Takeuchi, D., Sue, S., Yeh, M. Return rates and outcomes from ethnicity-specific mental health programs in Los Angeles. American Journal of Public Health 1995; 85:638-643.
6. Goncalves, M., Cook, B., Mulvaney-Day, N., Kinrys, G., Alegría, $M$. Retention in mental health care of Portuguese speaking patients. Transcultural Psychiatry 2013; 50(1):92-107.

7. Gaskell, G. Entrevistas individuais e grupais. In: Bauer, M.W., Gaskell, G., editors. Pesquisa qualitativa com texto, imagem e som: Um manual prático. Pétropolis. Editora Vozes; 2002.

8. Mayring, P. Qualitative Inhaltsanalyse: Grundlagen und Techniken. Weinheim: Dt. Studien-Verlag; 2000.

9. Bardin, L. Análise de conteúdo. Lisboa: Edições 70; 2009.

10. American Psychological Association. Ethical principles of psychologist and code of conduct. 2010. Available from http://www.apa.org/ ethics/code/index.aspx. 